\title{
Multi-wavelength properties of the high-energy bright Seyfert 1 galaxy IGR J18027-1455
}

\author{
J. A. Combi ${ }^{1}$, M. Ribó ${ }^{2,3}$, J. Martí $^{1}$, and S. Chaty ${ }^{3}$ \\ ${ }^{1}$ Departamento de Física (EPS), Universidad de Jaén, Campus Las Lagunillas s/n, 23071 Jaén, Spain \\ e-mail: jcombi@ujaen.es \\ 2 Departament d'Astronomia i Meteorologia, Universitat de Barcelona, Martí i Franquès 1, 08028 Barcelona, Spain \\ 3 AIM - Astrophysique Interactions Multi-échelles (UMR 7158 CEA/CNRS/Université Paris 7 Denis Diderot), CEA Saclay, \\ DSM/DAPNIA/Service d'Astrophysique, Bât. 709, L'Orme des Merisiers, 91191 Gif-sur-Yvette Cedex, France \\ Received 7 April 2006 / Accepted 8 August 2006
}

\section{ABSTRACT}

\begin{abstract}
A new sample of hard X-ray sources in the Galactic Plane is being revealed by the regular observations performed by the INTEGRAL satellite. The full characterization of these sources is mandatory to understand the hard X-ray sky. Here we report new multifrequency radio, infrared and optical observations of the source IGR J18027-1455, as well as a multi-wavelength study from radio to hard X-rays. The radio counterpart of IGR J18027-1455 is not resolved at any observing frequency. The radio flux density is well fitted by a simple power law with a spectral index $\alpha=-0.75 \pm 0.02$. This value is typical of optically thin non-thermal synchrotron emission originated in a jet. The NIR and optical spectra show redshifted emission lines with $z=0.034$, and a broad H $\alpha$ line profile with $F W H M \sim 3400 \mathrm{~km} \mathrm{~s}^{-1}$. This suggests an Active Galactic Nucleus (AGN) of type 1 as the optical counterpart of IGR J18027-1455. We confirm the Seyfert 1 nature of the source, which is intrinsically bright at high energies both in absolute terms and when scaled to a normalized $6 \mathrm{~cm}$ luminosity. Finally, comparing its X-ray luminosity with isotropic indicators, we find that the source is Compton thin and AGN dominated. This indicates that INTEGRAL might have just seen the tip of the iceberg, and several tens of such sources should be unveiled during the course of its lifetime.
\end{abstract}

Key words. X-rays: individuals: IGR J18027-1455 - X-rays: galaxies - radio continuum: galaxies - galaxies: Seyfert

\section{Introduction}

Unidentified high energy sources have been a subject of interest from the early days of the COS-B era. In the 1990s, with the advent of X-ray $/ \gamma$-ray satellites like $A S C A$ and $C G R O$ the number of sources with unidentified counterparts at other frequencies increased considerably. During the first year of observations, the IBIS/ISGRI instrument on board the INTEGRAL satellite (Winkler et al. 2003) detected 123 hard X-ray/ $\gamma$-ray point sources, 28 of which had no clear identification with known objects in other ranges of the electromagnetic spectrum (Bird et al. 2004). These X-ray/ $\gamma$-ray emitters could be high or low mass X-ray binaries, radio quiet pulsars, clusters of galaxies, or a significant fraction of any class of AGNs heavily obscured, at few $\mathrm{keV}$, by the absorbing material of the galactic plane. The possibility that several unidentified IBIS sources were of extragalactic nature was early suggested by some authors (Ribó et al. 2004; Combi et al. 2004; Masetti et al. 2004a; Masetti et al. 2004b; Bassani et al. 2004; Combi et al. 2005).

The source IGR J18027-1455 is one of such sources. It was discovered in the energy range from 20 to $100 \mathrm{keV}$ during $769 \mathrm{ks}$ of observations. Looking for possible counterparts Combi et al. (2004) found two weak point-like radio sources from the $20 \mathrm{~cm}$ NRAO VLA Sky Survey (NVSS, Condon et al. 1998) inside its 2 arcmin-radius position error circle (see Fig. 1). One of them, NVSS J180247-145451, lies inside and near the edge of the $2 \sigma$ position error circle of the faint ROSAT X-ray source 1RXS J180245.5-145432 (Voges et al. 2000), which is the only soft X-ray source well within the IBIS/ISGRI error circle. In addition, inside the $2 \sigma$ position error ellipse of this radio source, it is located an extended near infrared (NIR) source,
2MASS J18024737-1454547 (Cutri et al. 2003; Skrutskie et al. 2006), with standard aperture magnitudes $J=12.78 \pm 0.01$, $H=11.52 \pm 0.01$, and $K_{\mathrm{s}}=10.72 \pm 0.01$. Its optical counterpart has average magnitudes $B=19.3 \pm 1.0, R=14.9 \pm 0.8$ and $I=13.8 \pm 0.5$ in the USNO-B1.0 catalog (Monet et al. 2003). The photometry of the NIR/optical counterpart is not consistent with a stellar spectrum (Combi et al. 2004). On the basis of spectroscopic optical observations Masetti et al. (2004b) have tentatively classified this source as a Seyfert 1 galaxy at redshift $z=0.035 \pm 0.001$.

An important characteristic of AGNs is that they radiate over a wide range of frequencies, from radio to gamma-rays. For this reason, multi-wavelength observations are an important tool to discriminate between objects of different classes. Here we report multi-wavelength observations of IGR J18027-1455 and discuss the obtained results. The structure of the paper is as follows. In Sect. 2 we describe our radio continuum, NIR and optical observations and present the results. In Sect. 3 we discuss the nature of all the detected multi-wavelength emissions and we summarize our conclusions in Sect. 4.

\section{Multi-wavelength observations and results}

\subsection{Radio}

The field of IGR J18027-1455 was observed with the NRAO ${ }^{1}$ Very Large Array (VLA) on 2004 December 4 and 9, with the

1 The National Radio Astronomy Observatory is a facility of the National Science Foundation operated under cooperative agreement by Associated Universities, Inc. 


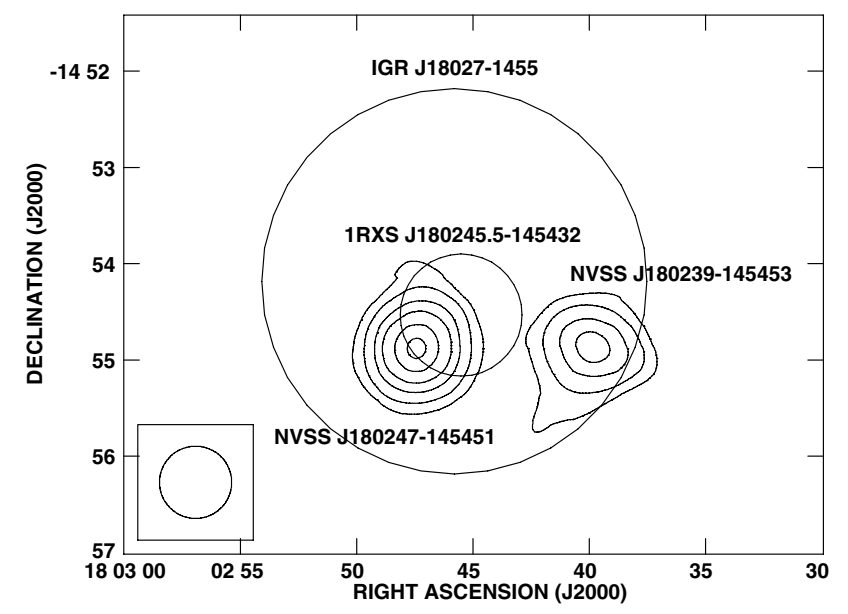

Fig. 1. Image of the NVSS data obtained with the VLA at $20 \mathrm{~cm}$ on 1997 October 13 around IGR J18027-1455. The image size is 7'.2×5'.5. Contours represent $-3,3,5,8,11,15,18$, and 22 times the rms noise level of $0.5 \mathrm{mJy}$ beam $^{-1}$. The circle in the bottom left corner represents the 45 arcsec of Full Width at Half Maximum $(F W H M)$ of the convolving beam. Two NVSS sources fall inside the $90 \%$ error circle in position of IGR J18027-1455, and one of them is within the $2 \sigma$ uncertainty error circle of a ROSAT source.

Table 1. Observational parameters of NVSS J180247-145451.

\begin{tabular}{cccc}
\hline \hline $\begin{array}{c}\lambda \\
(\mathrm{cm})\end{array}$ & $\begin{array}{c}\text { Flux density } \\
(\mathrm{mJy})\end{array}$ & $\begin{array}{c}\text { Apparent deconvolved angular size } \\
\left({ }^{\prime \prime} \times{ }^{\prime \prime}\right)\end{array}$ & $\begin{array}{c}\text { PA } \\
\left({ }^{\circ}\right)\end{array}$ \\
\hline 20 & $7.5 \pm 0.3$ & $(3.0 \pm 0.1) \times(2.1 \pm 0.1)$ & $95_{-6}^{+5}$ \\
6 & $2.8 \pm 0.2$ & $(0.94 \pm 0.03) \times(0.27 \pm 0.05)$ & $90_{-2}^{+2}$ \\
3.5 & $2.0 \pm 0.1$ & $(0.56 \pm 0.03) \times(0.27 \pm 0.04)$ & $102_{-4}^{+4}$ \\
\hline
\end{tabular}

array being in its most extended A configuration. Each observing run lasted for $2 \mathrm{~h}$ distributed among the wavelengths of 20 , 6 and $3.5 \mathrm{~cm}$, always with two IF pairs of $50 \mathrm{MHz}$ each. The visibilities were edited and calibrated using the AIPS software package of NRAO. The amplitude scale was set by observing the primary VLA calibrator 3C 286 and following the prescriptions recommended in the AIPS Cookbook concerning baseline range and reduction percentage of expected flux density as a function of the observed wavelength and array configuration. The phase calibrator observed was the nearby source J1733-130 at all frequencies. The analysis of our two individual epochs did not reveal radio sources with significant variability above the uncertainty of our amplitude calibration. Therefore, we concatenated the visibility data of individual epochs in order to analyze them together. Unfortunately, the source was accidentally placed 1'.8 from the phase center, so we had to correct for primary beam response using the AIPS task PBCOR. The resulting flux densities of NVSS J180247-145451, our candidate radio counterpart to IGR J18027-1455, are presented in Table 1.

Our first VLA maps of NVSS J180247-145451 displayed a clearly elongated radio source, as evidenced by the apparent deconvolved angular sizes of Table 1 . However, this elongation is compatible with the expected bandwidth smearing for a source located at 1'.8 from the phase center (Taylor et al. 2004). In fact, the object was found to be consistent with being unresolved at all frequencies. In order to avoid showing a visually misleading figure, we present our VLA map in Fig. 2 with the clean components convolved with a Gaussian beam artificially broadened in order to approximately compensate for the expected effects of bandwidth smearing. The corresponding beam at $6 \mathrm{~cm}$

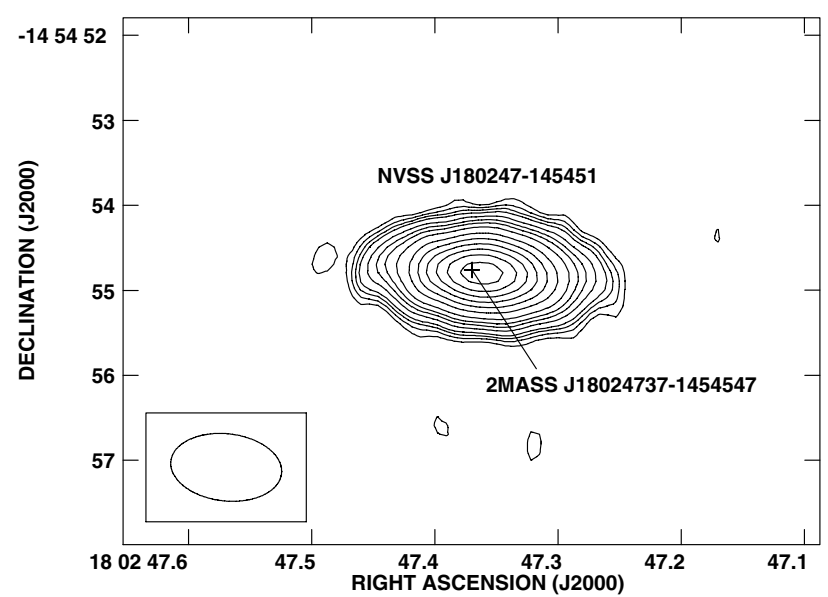

Fig. 2. Naturally weighted map of NVSS J180247-145451 obtained with the VLA in A configuration on December 2004 at $6 \mathrm{~cm}$. At this resolution the source has clearly a point-like appearance. The image size is $8^{\prime \prime} \times 6^{\prime \prime}$. The contours shown are $-3,3,4,5,6,8,10,12,15,20$, $25,30,35,40$ and 45 times $32 \mu \mathrm{Jy}_{\text {beam }}{ }^{-1}$, the rms noise. The ellipse shown at the bottom left corner corresponds to a beam of 1 '. $31 \times 0$ !' 79 , with position angle of $83^{\circ} .6$. The central cross marks the location of the proposed 2MASS near infrared counterpart ( $3 \sigma$ uncertainty).

wavelength is $1^{\prime \prime} 31 \times 0.79$ with position angle of $83^{\circ} .6$, which reveals the point-like nature of the source at this resolution.

Our best estimate of the radio source position is $\alpha_{\mathrm{J} 2000.0}=$ $18^{\mathrm{h}} 02^{\mathrm{m}} 47^{\mathrm{s}} .375 \pm 0.003$ and $\delta_{\mathrm{J} 2000.0}=-14^{\circ} 54^{\prime} 54^{\prime \prime} .78 \pm 00^{\prime} 02$, determined from $3.5 \mathrm{~cm}$ observations. The positions of the objects at different wavelengths are summarized in Table 2. The radio spectrum is well fitted by a simple power law $S_{v}=$ $(9.7 \pm 0.3 \mathrm{mJy})(v / \mathrm{GHz})^{-0.75 \pm 0.02}$.

\subsection{Near to far infrared}

We conducted photometric $\left(J, H\right.$ and $K_{\mathrm{s}}$ filters) and spectroscopic $(0.9-2.5 \mu \mathrm{m})$ NIR observations of 2MASS J18024737-1454547 on 2004 July 10 with the spectro-imager SofI, installed on the ESO New Technology Telescope (NTT). We used the large field imaging of SofI's detector, giving an image scale of $0^{\prime} .288$ pixel $^{-1}$ and a field of view of $4.94 \times 4.94$. Concerning the photometric observations, we repeated a set of observations for each filter with 9 different $30^{\prime \prime}$ offset positions including 2MASS J18024737-1454547, with an integration time of $90 \mathrm{~s}$ for each exposure, following the standard jitter procedure allowing to cleanly subtract the blank sky emission in NIR. We observed two photometric standard stars of the faint NIR standard star catalog of Persson et al. (1998): sj9157 and sj9172.

We used the IRAF (Image Reduction and Analysis Facility package) suite to perform the data reduction, including flatfielding and NIR sky subtraction. For the three obtained images, one in each filter, we obtained an astrometric solution by using more than 200 coincident 2MASS objects, with a final rms of 0.07 in each coordinate. We show the final $K_{\mathrm{s}}$ band image in Fig. 3, where the extended nature of 2MASS J18024737-1454547 can be easily seen. We carried out aperture photometry and transformed the instrumental magnitudes into apparent magnitudes with the standard relation: $\operatorname{mag}_{\text {app }}=\operatorname{mag}_{\text {inst }}-\mathrm{Zp}-$ ext $\times A M$ where $\operatorname{mag}_{\text {app }}$ and mag $\operatorname{minst}_{\text {ins }}$ are respectively the apparent and instrumental magnitudes, $\mathrm{Zp}$ is the zero-point, ext the extinction and $A M$ the airmass. The observations were performed through an airmass close to 1 . 
Table 2. Equatorial coordinates and position uncertainties of the sources discussed in the text.

\begin{tabular}{|c|c|c|c|c|}
\hline Source & $\alpha_{\text {(J2000.0 ICRS })}$ & 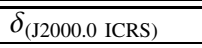 & Pos. uncertainty & Astrometry \\
\hline IGR J18027-1455 & $18^{\mathrm{h}} 02^{\mathrm{m}} 46^{\mathrm{s}} .0$ & $-14^{\circ} 54^{\prime} 10^{\prime} 0$ & $2^{\prime}(90 \%$ or $1.6 \sigma)$ & 1st IBIS/ISGRI (Bird et al. 2004) \\
\hline NVSS J180247-145451 & $18^{\mathrm{h}} 02^{\mathrm{m}} 47^{\mathrm{s}} .375$ & $-14^{\circ} 54^{\prime} 54^{\prime \prime} \cdot 78$ & $0 . \prime 02(68 \%$ or $1 \sigma)$ & this work \\
\hline 2MASS J18024737-1454547 & $18^{\mathrm{h}} 02^{\mathrm{m}} 47^{\mathrm{s}} .370$ & $-14^{\circ} 54^{\prime} 54^{\prime \prime} \cdot 76$ & $0.03(68 \%$ or $1 \sigma)$ & 2MASS (Cutri et al. 2003) \\
\hline USNO-B1.0 0750-0506536 & $18^{\mathrm{h}} 02^{\mathrm{m}} 47^{\mathrm{s}} .38$ & $-14^{\circ} 54^{\prime} 55^{\prime \prime} .1$ & $0.2(68 \%$ or $1 \sigma)$ & USNO-B1.0 (Monet et al. 2003) \\
\hline 1RXS J180245.5-145432 & $18^{\mathrm{h}} 02^{\mathrm{m}} 45^{\mathrm{s}} .5$ & $-14^{\circ} 54^{\prime} 32^{\prime \prime} 0$ & $19^{\prime} 0(68 \%$ or $1 \sigma)$ & ROSAT (Voges et al. 2000) \\
\hline
\end{tabular}

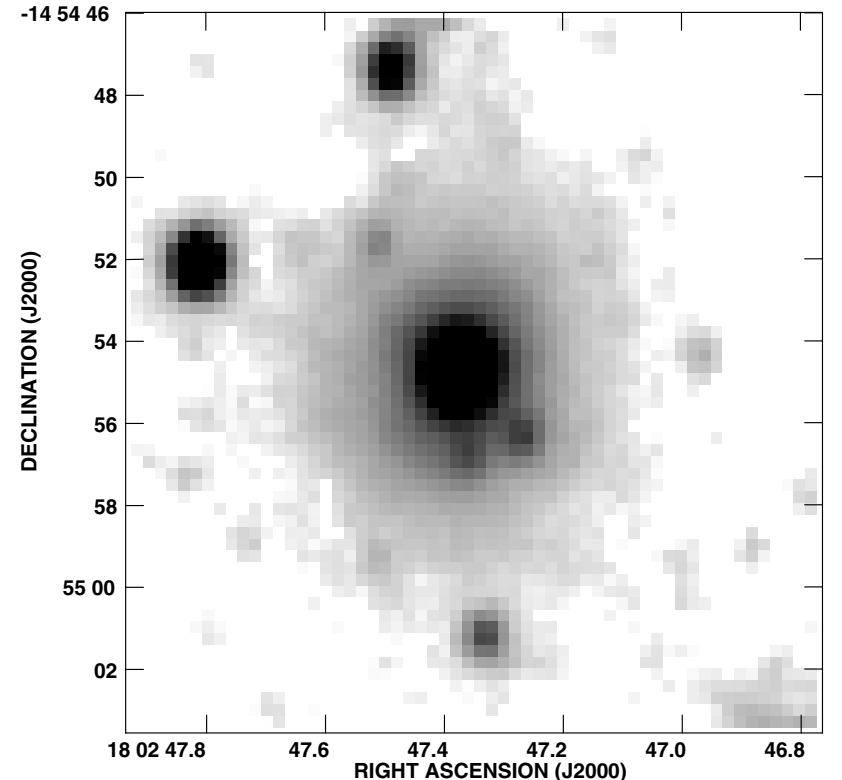

Fig. 3. $K_{\mathrm{s}}$ band image of the region around 2MASS J18024737-1454547 obtained with the NTT on 2004 July 10. A square root transfer function has been used to enhance the faint emission. The extended nature of the NIR source, the host galaxy, is clearly visible, mostly aligned in the north-south direction. The image size is $17^{\prime \prime} \times 18^{\prime \prime}$.

We obtained two sets of measurements for different appertures to include only the nucleus or the nucleus plus all the extended emission. Using a 4-pixel aperture diameter (1".2), and an adjacent annulus with outer radius of 5 pixels to estimate the sky background, we obtain: $J=14.9 \pm 0.1, H=13.3 \pm 0.1$ and $K_{\mathrm{s}}=11.7 \pm 0.1$. The magnitudes of the whole extended emission (nucleus+host galaxy) are: $J=12.77 \pm 0.02, H=11.41 \pm 0.03$ and $K_{\mathrm{s}}=10.44 \pm 0.04$. These last values are compatible within errors to those present in the 2MASS catalog, obtained with a 4 " aperture, except for the $K_{\mathrm{S}}$ band, where we find a slightly brighter source.

Concerning the NIR spectroscopy, we took 12 spectra with the Blue and Red grisms, respectively. The position of 2MASS J18024737-1454547 in the slit was offset $30^{\prime \prime}$ in half of the exposures to subtract the blank NIR sky. The total integration time was $240 \mathrm{~s}$ in each grism. We took Xe lamp exposures to perform the wavelength calibration. We extracted the spectra using the IRAF NOAO TWODSPEC package. Figure 4 shows the obtained normalized spectrum with the most important NIR emission lines indicated. We were unable to completely remove the telluric features between 1.8 and $1.93 \mu \mathrm{m}$, due to the atmospheric absorption. Several lines as $\mathrm{Pa} \delta$, He I $\lambda 10830$, O I $\lambda 11287$ (clearly detected after binning the spectrum), $\mathrm{Pa} \beta$ and $\mathrm{Pa} \alpha$ are clearly visible. Using these NIR emission lines we obtain a redshift to the source of $z=0.034 \pm 0.001$. We found no Br $\gamma$ emission nor any $[\mathrm{Fe} \mathrm{II}]$ component.

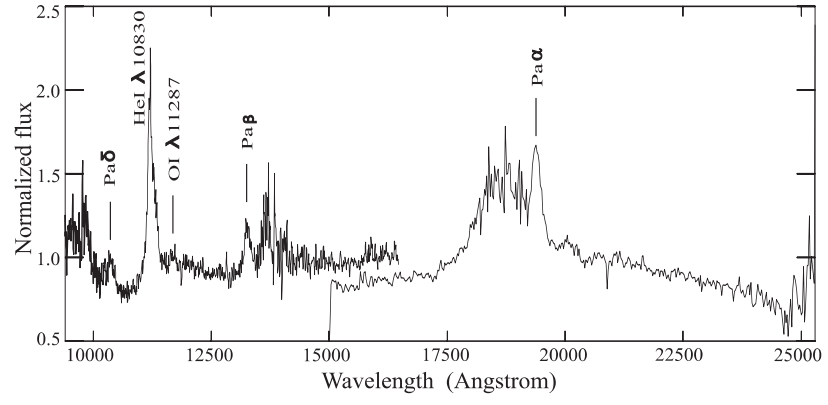

Fig. 4. Near infrared spectrum of 2MASS J18024737-1454547, the counterpart of IGR J18027-1455, acquired with the NTT on 2004 July 10. The identified emission lines are indicated. A redshift of $z=0.034 \pm 0.001$ is obtained. The strong noise redwards of $\mathrm{Pa} \beta$ and bluewards of $\mathrm{Pa} \alpha$ is due to telluric features.

We finally cross checked the position of the radio source NVSS J180247-145451 with the IRAS ${ }^{2}$ Point Source cata$\log$. At the mid-far infrared part of the spectrum, from 12 to 100 microns, we found no counterpart to our target. A total of 1168 IRAS sources lie in a circle of $3^{\circ}$ radius centered around the radio position. As explained in Filliatre \& Chaty (2004), we estimate as a robust upper limit for our target the flux such as $90 \%$ of the IRAS sources have greater fluxes, leading to 0.40 , $0.35,0.72,9.1 \mathrm{Jy}$ at $12,25,60$ and 100 microns, respectively.

\subsection{Optical}

The optical counterpart candidate of IGR J18027-1455, namely USNO-B1.0 0750-0506536, was observed in 2004 July 19 with the $2.2 \mathrm{~m}$ telescope of the Centro Astronómico Hispano Alemán (CAHA) in Calar Alto (Spain) under Director Discretionary Time (DDT). We used the CAFOS spectrograph with the grism R400 initially selected based on sensitivity criteria. This instrumental setup provided a dispersion of $9.65 \AA$ pixel $^{-1}$ with the CCD detector being a SITE\#1d_15 chip. Three science exposures, of $20 \mathrm{~min}$ each, were taken and combined into a single spectrum. The frames were bias subtracted, flatfielded and wavelength calibrated using $\mathrm{HgCd} / \mathrm{He} / \mathrm{Rb}$ lamps and the IRAF software package. No flux calibration was obtained during our DDT observations and, therefore, the resulting spectrum in Fig. 5 is presented in normalized units. The optical spectrum is strongly dominated by $\mathrm{H} \alpha$ emission with $z=0.034 \pm 0.001$ and $\mathrm{a}$ broad profile with a $F W H M \simeq 3400 \pm 300 \mathrm{~km} \mathrm{~s}^{-1}$ (mean between Gaussian and Lorentzian fits without deblending of the [N II] lines).

\section{Discussion}

The position agreement between the IGR J18027-1455, 1RXS J180245.5-145432 and NVSS J180247-145451

\footnotetext{
${ }^{2}$ http://irsa.ipac.caltech.edu/applications/Gator/
} 


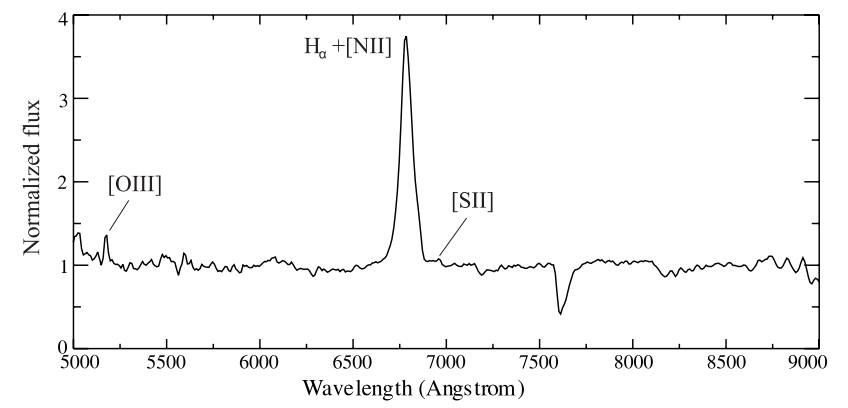

Fig. 5. Averaged optical spectrum of the optical counterpart of IGR J18027-1455 acquired with CAFOS on the $2.2 \mathrm{~m}$ telescope at CAHA on 2004 July 19. The spectrum has been smoothed with a Gaussian filter. The identified emission lines are indicated. A broad $\mathrm{H} \alpha$ line dominates the spectrum. A redshift of $z=0.034 \pm 0.001$ is obtained.

indicates that these are the multi-wavelength manifestations of the same source radiating at different bands of the electromagnetic spectrum. Moreover, the recently published second IBIS/ISGRI catalog (Bird et al. 2006) lists a position and error circle for IGR J18027-1455 that clearly exclude the other radio source, namely NVSS J180239-145453, as a possible counterpart (even at the $90 \%$ confidence level). The NIR source 2MASS J18024737-1454547 has a position in agreement within errors with our new precise radio position of NVSS J180247-145451. Its optical counterpart is USNOB1.0 0750-0506536. Both the NIR and optical objects are clearly extended (this work and Masetti et al. 2004b, respectively). In addition, the redshifted emission lines seen in the NIR and optical spectra reveal an extragalactic object at a redshift of $z=0.034 \pm 0.001$, with a broad $\mathrm{H} \alpha$ emission line with $F W H M \simeq 3400 \pm 300 \mathrm{~km} \mathrm{~s}^{-1}$ (these values are compatible with those of Masetti et al. 2004b, although they reported a slightly narrower emission line with $F W H M \sim 2700 \mathrm{~km} \mathrm{~s}^{-1}$ ). We are therefore seeing the broad line region (BLR), and the object is classified as a type 1 AGN.

The steep radio spectral index $(\alpha=-075 \pm 0.02)$ strongly supports a non-thermal emission mechanism of synchrotron nature. This is clearly compatible with optically thin extended jet emission from an extragalactic source. On the other hand, the NVSS flux density of the source at $1.4 \mathrm{GHz}$ is $10.5 \pm 0.6 \mathrm{mJy}$, to be compared with our measurement of $7.5 \pm 0.3 \mathrm{mJy}$. Both values are only marginally consistent at the $3 \sigma$ level, suggesting that the source is variable at radio wavelengths. We note that $0.61 \mathrm{GHz}$ ( $49 \mathrm{~cm}$ wavelength) observations conducted 3 months later provided a detection at a level of $5.0 \pm 0.35 \mathrm{mJy}$ (Pandey et al. 2006), either supporting the variability of the source or indicating that there is a low frequency turnover.

The NIR spectrum is very similar to other well studied Seyfert 1 galaxies such as NGC 863 or Mrk 335 (Rodríguez-Ardila et al. 2002), although the poor signal-to-noise ratio of our NIR observations is not enough to discriminate weak lines as in these cases. It is interesting to note that the permitted O I $\lambda 11287$ line is a feature completely associated with the BLR of Seyfert galaxies. In our case this line is marginally detected, as in NGC 863 (Rodríguez-Ardila et al. 2002). The non-detection of the Bry line seems to suggest that thermal UV heating is not important, as it also happens in the case of NGC 1097 (Reunanen et al. 2002).

The multiwavelength properties of IGR J18027-1455 strongly support an AGN nature and more specifically a type 1 Seyfert galaxy. In order to compare the broadband emission of the object with that one of the mean for type 1 Seyfert galaxies, we have determined the nuclear spectral energy distribution (SED), from the radio to the gamma-ray band. The observations used to build the SED have been discussed in Sect. 2. The observed magnitudes in the NIR and optical bands were corrected for reddening from Galactic extinction based on the estimated hydrogen column density $N_{\mathrm{H}}=(5.0 \pm 1.0) \times 10^{21} \mathrm{~cm}^{-2}$ (Dickey \& Lockman 1990) and the Predehl \& Schmitt (1995) relationship $A_{\mathrm{V}}=(5.59 \pm 0.10) \times 10^{-22} N_{\mathrm{H}}$, which gives $A_{\mathrm{V}}=2.8 \pm 0.6 \mathrm{mag}$. The transformation between the absorption in the optical $A_{\mathrm{V}}$ and that at other wavelengths was computed according to the Rieke \& Lebofsky (1985) interstellar extinction law.

At soft X-ray energies, between $0.1-2.4 \mathrm{keV}$, the flux was obtained using the ROSAT/PSPC count rate of $(3.25 \pm 1.39) \times$ $10^{-2}$ count $^{-1}$ (Voges et al. 2000) and a photon index of $\Gamma=$ $+1.9 \pm 01$, typical of Seyfert 1 galaxies (Malizia et al. 2003). We used the web based tool PIMMS v3.7 $\mathrm{a}^{3}$ and the $N_{\mathrm{H}}$ value given above to obtain an unabsorbed flux of $2.4_{-1.3}^{+2.0} \times 10^{-12} \mathrm{erg} \mathrm{cm}^{2} \mathrm{~s}^{-1}$ (propagating all possible uncertainties). In addition, extrapolation of the ROSAT/PSPC count rate with the same photon index and absorption, considering all possible uncertainties, provides a flux of $1.5_{-0.9}^{+1.4} \times 10^{-12} \mathrm{erg} \mathrm{cm}^{-2} \mathrm{~s}^{-1}$ in the $2-10 \mathrm{keV}$ energy range.

The average fluxes detected by INTEGRAL in the 20-40 and $40-100 \mathrm{keV}$ energy ranges are $3.0 \pm 0.2$ and $3.3 \pm 0.3 \mathrm{mCrab}$, respectively (Bird et al. 2006). These can be converted to the cgs fluxes (assuming a Crab-like spectrum and the values in Bird et al. 2006) $(2.3 \pm 0.2) \times 10^{-11} \mathrm{erg} \mathrm{cm}^{2} \mathrm{~s}^{-1}$ and $(3.1 \pm 0.3) \times$

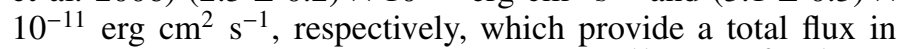
the $20-100 \mathrm{keV}$ range of $(5.4 \pm 0.4) \times 10^{-11} \mathrm{erg} \mathrm{cm}^{2} \mathrm{~s}^{-1}$. We note that an analysis with more INTEGRAL data reveals the following values in the $20-100 \mathrm{keV}$ range (Bassani et al. 2006): $2.6 \pm 0.1 \mathrm{mCrab}$ and $(4.4 \pm 0.2) \times 10^{-11} \mathrm{erg} \mathrm{cm}^{2} \mathrm{~s}^{-1}$. As can be seen there are hints of variability in the hard X-ray/gamma-ray flux of IGR J18027-1455, and the average of $(4.9 \pm 0.5) \times$ $10^{-11} \mathrm{erg} \mathrm{cm}^{2} \mathrm{~s}^{-1}$ will be used when plotting the SED.

To compute the monochromatic luminosities we have adopted the cosmological parameters from Spergel et al. (2003): $H_{0}=71 \mathrm{~km} \mathrm{~s}^{-1} \mathrm{Mpc}^{-1}, \Omega_{\Lambda}=0.73$ and $\Omega_{\mathrm{m}}=0.27$. Using our measured redshift of $z=0.034 \pm 0.001$ we obtain ${ }^{4}$ a luminosity distance of $147 \pm 5 \mathrm{Mpc}$ for IGR J18027-1455, leading to a hard $\mathrm{X}$-ray luminosity of $(1.3 \pm 0.1) \times 10^{44} \mathrm{erg} \mathrm{s}^{-1}$. The source is one of brightest Seyfert 1 galaxies detected so far: it is brighter than any of the Seyfert 1 galaxies detected with BeppoSAX (Panessa 2004), and the 4th brightest one among the 14 detected with INTEGRAL (Bassani et al. 2006).

We show in Fig. 6 the overall nuclear SED of IGR J18027-1455 in a $\log (v)-\log \left(v L_{v}\right)$ representation, but normalized at $6 \mathrm{~cm}$ for comparison with the mean SEDs of Seyfert galaxies (Panessa 2004; Panessa et al. 2004). The real luminosities of IGR J18027-1455 are 1.21 dex lower than those shown. Although in the NIR domain we have used nuclear magnitudes, the optical magnitudes ( $I$ and $B$ from USNO-B1.0 and $R=16.55 \pm 0.01$ from Masetti et al. 2004b) could be strongly contaminated by the host galaxy due to a limited angular resolution of $\sim 1^{\prime \prime}$ pixel ${ }^{-1}$. We have not plotted the extrapolated $2-10 \mathrm{keV}$ flux. Although the archival IRAS data does not allow us to detect the far infrared bump, the NIR/ROSAT data clearly show a SED typical of Seyfert 1 galaxies. However, the INTEGRAL data show that the source is clearly high-energy bright. Therefore, IGR J18027-1455 is

\footnotetext{
3 http://heasarc.gsfc.nasa.gov/Tools/w3pimms.html

4 http://www . astro.ucla. edu/ wright/CosmoCalc.html
} 


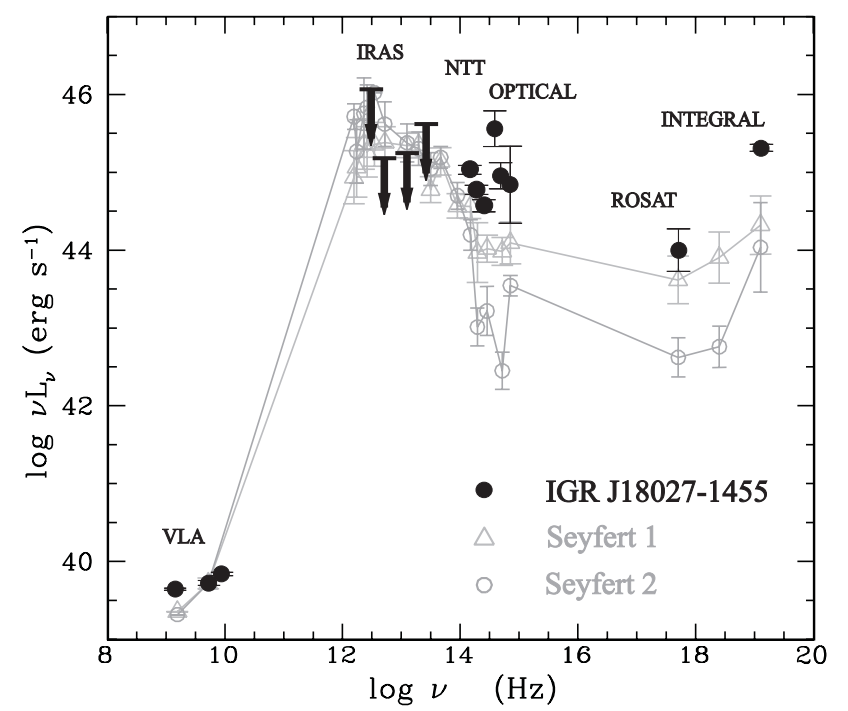

Fig. 6. Overall nuclear spectral energy distribution of IGR J18027-1455 (filled symbols) from the radio to the hard $\mathrm{X}$-ray/gamma-ray band. Optical stands for the $I$ and $B$ USNO-B1.0 and $R$ magnitude from Masetti et al. (2004b). The SED has been normalized at $6 \mathrm{~cm}$ (assuming $\left.\log \left(v L_{\nu}\left[\mathrm{erg} \mathrm{s}^{-1}\right]\right)=39.75\right)$ in order to compare between type 1 and type 2 Seyfert galaxies (open symbols; adapted from Panessa 2004; vertical bars are errors of the averages, not the standard deviation of the samples). The real luminosities of our target source are 1.21 dex lower than those shown. The SED of IGR J18027-1455 resembles the average one for Seyfert 1 galaxies, although it is brighter in the hard X-ray domain. Optical data is probably contaminated by the host galaxy.

not only among the brightest type 1 Seyfert galaxies at high energies in absolute terms, but also when normalized to the $6 \mathrm{~cm}$ luminosity.

In the basic scheme for unification of AGNs, Seyfert galaxies are divided in two class. Those that have narrow forbidden lines and a BLR in their optical spectrum (Seyfert 1) and those that only have narrow lines (Seyfert 2 ). While the broad lines originate near the central massive black hole located at $\leq 0.1 \mathrm{pc}$, narrow lines arise far from the nuclear engine at a distance $\leq 100 \mathrm{pc}$. Specifically they are the same type of object but, according to the standard model, in Seyfert 2 galaxies the BLR is obscured by a molecular torus (Antonucci 1993). For this reason, the majority of these objects are Compton thick, that is, the medium is thick to Compton scattering so that the transmitted component is dramatically suppressed below $10 \mathrm{keV}$ down to the NIR domain.

We can thus further check the Seyfert 1 nature of IGR J18027-1455 and its agreement with unification schemes by comparing its soft X-ray luminosity with isotropic indicators. This allows us to discriminate if starburst or AGN is the dominant component, and at the same time to assess if the source is Compton thin or Compton thick. If the presence of a molecular torus around the central region is important, the X-ray emission coming from the central engine will be negligible and it should be coming from a more extended zone like the NLR or a starburst region. In this case, the column density could be obtained indirectly from the flux ratio between the X-ray flux and isotropic emission measurements like the [O III $] \lambda 5007$ and far-infrared fluxes. Both are good isotropic indicators, while [O III $] \lambda 5007$ emission is produced by photons originated in the central nucleus, infrared emission is mainly associated to starforming activity, and therefore produced in a larger region than that of the molecular torus.
To compute the [O III] $\lambda 5007$ flux we have used our normalized optical spectrum and the average optical spectrum of Masetti et al. (2004b). The line is clearly detected at $\lambda=5175 \AA$. Smoothing our spectrum with a Gaussian function, its equivalent width is $10 \pm 2 \AA$ and the [O III] $\lambda 5007$ flux is $7.1 \times$ $10^{-16} \mathrm{erg} \mathrm{cm}^{-2} \mathrm{~s}^{-1}$. This flux has been corrected for extinction using the equation given by Bassani et al. (1999). Using a $\mathrm{H} \alpha \cong 8.5 \times 10^{-16} \mathrm{erg} \mathrm{cm} \mathrm{cm}^{-2} \mathrm{~s}^{-1} \AA^{-1}$ and a $\mathrm{H} \beta \leq 0.5 \times$ $10^{-16} \mathrm{erg} \mathrm{cm}^{-2} \mathrm{~s}^{-1} \AA^{-1}$, the observed flux ratio $\mathrm{H} \alpha / \mathrm{H} \beta \geq 17$ and $F_{\text {[OIII], cor }} \geq 1.2 \times 10^{-13} \mathrm{erg} \mathrm{cm}^{-2} \mathrm{~s}^{-1}$. Since the $F_{\mathrm{X}}$, between $2-10 \mathrm{keV}$, is $0.6-2.9 \times 10^{-12} \mathrm{erg} \mathrm{cm}^{-2} \mathrm{~s}^{-1}$ the $F_{\mathrm{X}} / F_{\text {[OIII] }}$ ratio is between 5 and 24 . According to the flux diagnostic diagrams introduced by Panessa (2004) for type 1 and 2 Seyferts, the source is Compton thin. To calculate the far-infrared flux we have adopted the equation of Mulchaey et al. (1994). As a result the infrared flux is $F_{\mathrm{IR}} \leq 8 \times 10^{-11} \mathrm{erg} \mathrm{cm}^{-2} \mathrm{~s}^{-1}$. Therefore, the flux ratio $F_{[\mathrm{OIII}]} / F_{\mathrm{IR}} \geq 1.5 \times 10^{-3}$. According to Panessa (2004), this shows that AGN contribution, not starburst, is the dominant component.

\section{Summary}

We can summarize our main results as follows:

1. The radio counterpart of IGR J18027-1455 has not been resolved at any frequency by the VLA in its most extended A configuration. The radio flux density is well fitted by a simple power law with a spectral index $\alpha=-0.75 \pm 0.02$, typical of optically thin synchrotron radiation originated by a non-thermal jet. VLBI observations are needed to resolve the expected jet-like structure of this high energy object.

2. The NIR spectrum of the IGR J18027-1455 counterpart shows several emission lines with a redshift $z=0.034 \pm$ 0.001 . The optical spectrum is strongly dominated by redshifted $\mathrm{H} \alpha$ emission with $z=0.034 \pm 0.001$ and a broad profile with $F W H M \simeq 3400 \pm 300 \mathrm{~km} \mathrm{~s}^{-1}$. This confirms that IGR J18027-1455 is a type 1 AGN.

3. We determined the nuclear SED of IGR J18027-1455 using new radio, optical and infrared observations and available data in the literature. Comparing our obtained SED with the average ones of Seyfert galaxies, we found that it is typical of Seyfert 1 galaxies, although brighter than the mean at high energies, both in absolute terms and when using a normalized $6 \mathrm{~cm}$ luminosity.

4. We checked independently the Seyfert 1 nature of IGR J18027-1455 by comparing its X-ray luminosity with isotropic indicators. The source is Compton thin, as expected in unification schemes, and the AGN contribution is the dominant component.

Acknowledgements. J.A.C. is a researcher of the programme Ramón y Cajal funded jointly by the Spanish Ministerio de Educación y Ciencia (MEC) and Universidad de Jaén. M.R. has been supported by the French Space Agency (CNES) and by a Marie Curie Fellowship of the European Community programme Improving Human Potential under contract number HPMF-CT-200202053, and is being supported by a Juan de la Cierva fellowship from MEC. The authors also acknowledge support by DGI of MEC under grants AYA200407171-C02-02 and AYA2004-07171-C02-01, FEDER funds and Plan Andaluz de Investigación of Junta de Andalucía as research group FQM322. Partly based on observations collected at the European Southern Observatory, Chile (observing proposal ESO N ${ }^{\circ}$ 073.D-0339), and on observations collected at the Centro Astronómico Hispano Alemán (CAHA) at Calar Alto, Spain, operated jointly by the Max-Planck Institut für Astronomie and the Instituto de Astrofísica de Andalucía (CSIC). 


\section{References}

Antonucci, R. 1993, ARA\&A, 31, 473

Bassani, L., Dadina, M., Maiolino, R., et al. 1999, ApJS, 121, 473

Bassani, L., Malizia, A., Stephen, J. B., et al. 2004, in Proceedings of The 5th INTEGRAL Workshop: The Integral Universe, ESA SP-552, 139 [arXiv:astro-ph/0404442]

Bassani, L., Molina, M., Malizia, A., et al. 2006, ApJ, 636, L65

Bird, A. J., Barlow, E. J., Bassani, L., et al. 2004, ApJ, 607, L33

Bird, A. J., Barlow, E. J., Bassani, L., et al. 2006, ApJ, 636, 765

Combi, J. A., Ribó, M., \& Mirabel, I. F. 2004, ATel, 246

Combi, J. A., Ribó, M., \& Mirabel, I. F. 2005, Ap\&SS, 297, 385

Condon, J. J., Cotton, W. D., Greisen, E. W., et al. 1998, AJ, 115, 1693

Cutri, R. M., Skrutskie, M. F., van Dyk, S., et al. 2003, VizieR Online Data Catalog, II/246 http: //cdsweb.u-strasbg.fr/viz-bin/Cat?II/246

Dickey, J. M., \& Lockman, F. J. 1990, ARA\&A, 28, 215

Filliatre, Ph., \& Chaty, S. 2004, ApJ, 616, 469

Malizia, A., Bassani, L., Stephen J. B., et al. 2003, ApJ, 589, L17

Masetti, N., Palazzi, E., Bassani, L., Malizia, A., \& Stephen, J. B. 2004a, ATel, 310

Masetti, N., Palazzi, E., Bassani, L., Malizia, A., \& Stephen, J. B. 2004b, A\&A, 426, L41

Monet, D. G., Levine, S. E., Canzian, B., et al. 2003, AJ, 125, 984 http://cdsweb.u-strasbg.fr/viz-bin/Cat?I/284
Mulchaey, J. S., Koratkar, A., Ward, M. J., et al. 1994, ApJ, 436, 586

Pandey, M., Manchanda, R. K., Rao, A. P., Durouchoux, P., \& Ishwara-Chandra 2006, A\&A, 446, 471

Panessa, F. 2004, Ph.D. Thesis, University of Bologna

Panessa, F., Bassani, L., Cappi, M., Dadina, M., \& Iwasawa, K. 2004, Proceedings of the VI Italian Conference on Active Galactic Nuclei [arXiv: astro-ph/0410083]

Persson, S. E., Murphy, D. C., Krzeminski, W., Roth, M., \& Rieke, M. J. 1998, AJ, 116, 2475

Predehl, P., \& Schmitt, J. H. M. M. 1995, A\&A, 293, 889

Reunanen, J., Kotilainen, J. K., \& Prieto, M. A. 2002, MNRAS, 331, 154

Ribó, M., Combi, J. A., \& Mirabel, I. F. 2004, ATel, 235

Rieke, G. H., \& Lebofsky, M. J. 1985, ApJ, 288, 618

Rodríguez-Ardila, A., Viegas, S. M., Pastoriza, M. G., \& Prato, L. 2002, ApJ, 579, 214

Skrutskie, M. F., Cutri, R. M., Stiening, R., et al. 2006, AJ, 131, 1163

Spergel, D. N., Verde, L., Peiris, H. V., et al. 2003, ApJS, 148, 175

Taylor, G. B., Ulvestad, J. S., \& Perley, R. A. 2004, The Very Large Array Observational Status Summary,

http://www.vla.nrao.edu/astro/guides/vlas/current/vlas.ps

Voges, W., Aschenbach, B., Boller, Th., et al. 2000, VizieR Online Data Catalog, IX/29 http: //cdsweb.u-strasbg.fr/viz-bin/Cat?IX/29

Winkler, C., Courvoisier, T. J.-L., Di Cocco, G., et al. 2003, A\&A, 411, L1 